\title{
Migraine and Medical Ramifications: A Comprehensive Overview Based on Observational Study Meta-Analyses
}

\author{
Weiwei Chen ${ }^{1 *}$, Wenqi Qian ${ }^{2}$, Lixian Zhong ${ }^{3}$ and Gongwei Jing ${ }^{4}$ \\ ${ }^{1}$ Department of Gastroenterology, The First People's Hospital of Zunyi (The Third Affiliated Hospital of Zunyi Medical \\ University), Zunyi, China, ${ }^{2}$ Department of Pharmacy, People's Hospital of Qiandongnan and Dong Autonomous Prefecture, \\ Kaili, China, ${ }^{3}$ Department of Gastroenterology, The First Affiliated Hospital, Jinan University, Guangzhou, China, ${ }^{4}$ Department \\ of Nuclear Medicine, The First People's Hospital of Zunyi (The Third Affiliated Hospital of Zunyi Medical University), Zunyi, \\ China
}

Purpose: An umbrella review was conducted for comprehensively evaluating previous review-based literature together with meta-analysis of observational investigations probing correlations between migraine and medical end-point ramifications in patients. The breadth and validity of these associations were assessed.

Methods: Multiple online scientific repositories (including PubMed, Medline, Embase, and Web of Science) were investigated (inception-August 2021) for related meta-analyses focusing on links between migraine and all possible health/medical ramification end-points. A summary effect size and 95\% Cls were determined for each identified study with such links. Heterogeneity and small-study influence traces were also evaluated. The AMSTAR 2 platform was employed for evaluating standards of methodology, together with objective criteria, for assessing the standards of datasets from each medical end-point scrutinized in this study.

Results: A total of 25 scientific reports comprising 10,237,230 participants for 49 meta-analyses of observational studies were selected. Among such 49 outcomes, 30 demonstrated statistical significance $(P<0.05)$. Significant associations were observed in multiple diseases, including cardiovascular/cerebrovascular, cerebral, pregnancy-related and metabolic disorders, other outcomes, and mortality.

Conclusion: The results showed that migraine increased the risk of 29 health outcomes, though lowered the risk of breast cancer. However, evidence quality was graded as high only for angina. The evidence quality of ischaemic stroke, stroke, MACCE, WMAs, and asthma was graded as moderate. All remaining 24 outcomes had an evidence grade of "weak."

Keywords: migraine, health, medical ramifications, umbrella review, meta-analysis

\section{INTRODUCTION}

Migraine is a highly prevalent, disabling, complex primary headache-based condition, typically manifesting itself due to hyper-excitability of the central nervous system (CNS) (1). Migraine is diagnosed through multiple bouts of cranial pain and associated with a myriad of neurological symptom presentations. A migraine event is typically structured in phases: premonitory, aura, 
headache, postdrome, and interictal (2). Basic science studies indicate that there may be common pathways in migraine and other types of headache, such as persistent post-traumatic headache (PPTH). However, recent findings from structural and functional neuroimaging studies have attempted to describe the brain architecture of PPTH, suggesting the involvement of different networks compared to migraine (3). Migraine imposes a significant burden on patients and a great economic cost for society. It has a prevalence ranging from 2.6 to $21.7 \%$, with a mean of $12 \%$, depending on the population surveyed (4). Among individuals within the 30-49 year age bracket, peak migraine prevalence ranges from 11 to $20 \%$ for women and $3-8 \%$ for men, suggesting that women suffer a greater burden of migraine symptoms and disability in comparison to men (5).

In addition to causing uncomfortable symptoms including paroxysmal headaches, nausea, vomiting, photophobia, and phonophobia, migraine could exacerbate risks for incurring other adverse health outcomes. For example, earlier studies suggested that migraine patients experienced elevated risks for incurring cardiovascular diseases, including ischaemic stroke (6), hemorrhagic stroke (7), myocardial infarction (MI) (8), and angina (9). This might be explained by a number of plausible mechanisms, such as endothelial dysfunction, cerebral hypoperfusion, systemic vasculopathy, and a hypercoagulable state (10-13). Recently, emerging body of evidence from scientific literature reported the associations between migraine and other diseases, including restless leg syndrome (RLS) (14), diabetes (15), irritable bowel syndrome (IBS) (16), retinal nerve fiber layer (RNFL) thickness reduction (17), sudden sensorineural hearing loss (SSNHL) (18), major depression, and panic disorder (19). However, the published studies focused on a single health-related outcome.

Consequently, this umbrella-review study was performed for providing a detailed assessment of previously published reviews/meta-analyses that focused on the interplay between migraine and multiple heath end points. We also assessed the breadth and validity of these associations. This work suggests that migraine has a major adverse impact on human health, and will help to raise awareness of migraine and improve the motivation to treat it.

\footnotetext{
Abbreviations: PPTH, persistent post-traumatic headache; CNS, central nervous system; MI, myocardial infarction; RLS, restless leg syndrome; IBS, irritable bowel syndrome; RNFL, retinal nerve fiber layer; SSNHL, sudden sensorineural hearing loss; PRISMA, preferred reporting items for systematic reviews and meta-analyses; CIs, confidence intervals; OR, odds ratio; RR, relative risk; $H R$, hazard ratio; $\mathrm{PR}$, prevalence ratio; $\mathrm{MD}$, mean difference; $\mathrm{SMD}$, standard mean difference; MACCE, major adverse cardiovascular and cerebrovascular events; CAD, cervical artery dissection; CIMT, carotid artery intima-media thickness; PI, pulsatility index; CVR, cerebrovascular responsiveness; $\mathrm{MBFV}$, mean blood flow velocity; IHD, ischaemic heart disease; WMAs, white-matter abnormalities; ILLs, infarct-like lesions; PE, preeclampsia; LBW, low birth weight; PTB, preterm birth; SGA, gestational age; LDL-C, low-density lipoprotein cholesterol; TC, cholesterol; TG, triglyceride; HDL-C, high-density lipoprotein cholesterol; POAG, primary open angle glaucoma; ADHD, attention-deficit/hyperactivity disorder; CVD, cardiovascular; CHD, coronary heart disease; ICHD, international classification of headache diseases; MA, migraine with aura; MO, migraine without aura.
}

\section{MATERIALS AND METHODS}

The umbrella review was conducted in line with the PRISMA (Preferred Reporting Items for Systematic Reviews and MetaAnalyses) regulations (20), following a protocol registered with PROSPERO in advance (CRD42021273782).

\section{Search Strategy}

The PubMed, Medline, Embase, and Web of Science were scrutinized (from repository inception date until August 2021) using "migraine" OR "headache" AND "meta-analysis" OR "systematic review" as search-terms. Furthermore, the references section for each selected article was manually scrutinized to identify potential missing meta-analyses from the initial search.

\section{Study Selection}

Two authors (WQ and GJ) independently searched the titles and abstracts of eligible articles, followed by full text examination. All differences were discussed and resolved by consensus. Any disagreements that could not be resolved through consensus were arbitrated by a third reviewer (LZ). Articles that met the following criteria were included:

1) Meta-analyses of observational studies that evaluated the associations of migraine with any health outcomes in humans,

2) The summary effect size, with $95 \%$ confidence intervals (CIs), were available.

Whenever a single meta-analysis of multiple health outcomes was performed in one article, each outcome was included separately. Whenever multiple meta-analyses reported an identical health outcome, the meta-analysis review publication containing the highest amount of studies was selected. Systematic reviews without meta-analyses were excluded. Additionally, articles with unavailable full text were excluded. Articles discussing the increased risk of migraines from other diseases were also excluded.

\section{Data Extraction}

WQ and GJ independently collected data using a pre-designed table containing the following parameters: outcomes, first-author and publication year, study quantity and study design, total participant quantity/cases, metric-type (OR, odds ratio; RR, relative risk; $\mathrm{HR}$, hazard ratio; $\mathrm{PR}$, prevalence ratio; $\mathrm{MD}$, mean difference; SMD, standard mean difference), estimated summary effect and 95\% confidence intervals, $P$-value for statistically significant level, $P$-value for $Q$-test, and $P$-value for Egger's test.

\section{Data Analysis}

All summary estimates and 95\% CIs were extracted directly from articles, the results being deemed to have statistical significance whenever $P<0.05$, with $P$ being collected through confidence interval using a reported method (21), whenever it was not listed in the article. The between-study heterogeneity was evaluated by the $I^{2}$ statistic and Cochran's Q-test. Publication bias was evaluated by the Egger regression asymmetry test. $P<0.1$ indicated statistically significant heterogeneity and publication 


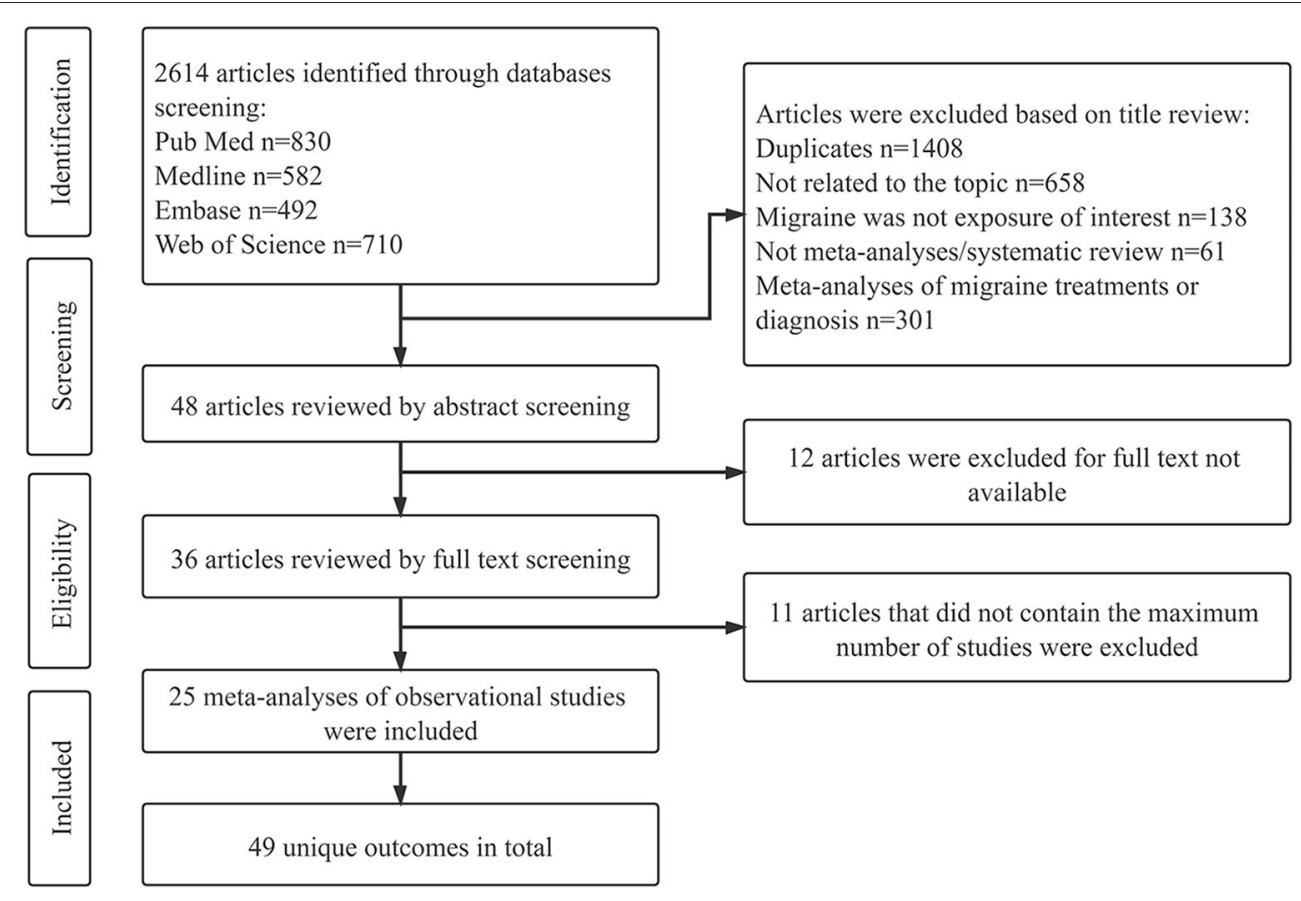

FIGURE 1 | Flowchart of study selection process for umbrella review.

bias. $I^{2}<25 \%$ was considered to be low heterogeneity, $I^{2}>75 \%$ was determined to be very high heterogeneity, with the remaining being classified as moderate-to-high heterogeneity.

\section{Evaluation of the Quality and Grading of Evidence}

AMSTAR2 (22) was applied for assessing standards in methodology within all selected investigations, deemed as robust and validated instruments involved in evaluating standards within previous systematic reviews and meta-analyses. The platform ranks the quality of a meta-analysis as critically low, low, moderate and high, based upon 16 pre-determined parameters. Regarding robustness for epidemiologic proof from each medical end-point, significant correlations $(P<0.05)$ were rated as high, moderate, or weak proof, in line with a grading exercise which was previously adopted within multiple research niches (23-25). The above evaluation process was independently completed by WQ and GJ.

\section{RESULTS}

\section{Search Results}

An in-depth flowchart for the selection protocol is illustrated in Figure 1. A total of 2,614 articles were initially identified from the four databases. 1,386 articles remained following duplicate removal, and 1,206 articles were removed from this study following scrutiny of publication title/abstract. Regarding the remaining 36 articles with full-text available, 11 were further excluded since such publications reported identical outcomes with other articles. Finally, 25 meta-analyses of observational studies, having 49 separate medical end-points were selected for this study.

All 25 articles were published between 2004 and 2021. The median quantity of meta-analyses investigation including observational investigations for each medical end-point was 12 (ranged 2-30). The median participant quantity was 313,908 (ranged 330-3,945,421), while the median case quantity was 1,793 (ranged 252-383,187) (Table 1). A vast array of medical end-points were listed: cardiovascular/cerebrovascular disorders $(n=22)$, imaging abnormalities $(n=3)$, pregnancy-linked conditions $(n=4)$, metabolic conditions $(n=4)$, other medical conditions $(n=12)$, and mortality $(n=4)$ (Figure 2$)$. From all 49 medical end-points, 30 reported effects had statistical significance $(P<0.05)$.

\section{Cardiovascular/Cerebrovascular Disorders}

The adverse effects of migraine on cardiovascular and cerebrovascular diseases are well-established. Migraine patient cohorts experienced elevated risk of ischaemic stroke (26), haemorrhagic stroke (27), stroke (8), major adverse cardiovascular and cerebrovascular events (MACCE) (8), angina (9), MI (9), and cervical artery dissection (CAD) (28). Such patient cohorts also experienced increased carotid artery intima-media thickness (CIMT), indicating links between atherosclerosis and migraine (29). The scrutinized meta-analyses also revealed that migraine-sufferers possibly have an increased pulsatility index (PI) and reduced cerebrovascular responsiveness (CVR) to posterior circulatory hypercapnia (30). Other findings included an elevated resting mean blood flow velocity (MBFV) within both anterior-and posterior-circulatory migraine sufferers 
TABLE 1 | Description of 49 meta-analyses of migraine and prevalence or incidence of diseases included in umbrella review.

\begin{tabular}{|c|c|c|c|c|c|c|c|c|c|c|c|}
\hline Outcomes & References & Number of studies & $\begin{array}{l}\text { Number of } \\
\text { participants }\end{array}$ & $\begin{array}{l}\text { Number of } \\
\text { cases }\end{array}$ & $\begin{array}{l}\text { Type of } \\
\text { metric }\end{array}$ & $\begin{array}{l}\text { Relative risk } \\
\qquad(95 \% \mathrm{CI})\end{array}$ & $\underset{\star}{\mathrm{P} \text { value }}$ & P value \# & $I^{2}(\%)$ & P value* & $\begin{array}{l}\text { Whether exist } \\
\text { publication bias }\end{array}$ \\
\hline \multicolumn{12}{|l|}{$\begin{array}{l}\text { Cardiovascular/cerebrovascular } \\
\text { disorders }\end{array}$} \\
\hline Ischemic stroke & Spector et al. (26) & $\begin{array}{l}8 \text { cohort studies, } \\
13 \text { case-control studies }\end{array}$ & 622,381 & 1,626 & OR & $2.04(1.72-2.43)$ & $<0.001$ & $<0.001$ & 63.5 & $0.66^{\&}$ & No \\
\hline Hemorrhagic stroke & Sacco et al. (27) & $\begin{array}{l}4 \text { cohort studies, } \\
4 \text { case-control studies }\end{array}$ & 316,989 & 91,914 & OR & $1.48(1.16-1.88)$ & 0.002 & 0.031 & 54.7 & 0.512 & No \\
\hline Stroke & Mahmoud et al. (8) & $\begin{array}{l}7 \text { cohort studies, } \\
6 \text { case-control studies }\end{array}$ & $1,033,338$ & 383,187 & $\mathrm{HR}$ & $1.42(1.25-1.61)$ & $<0.001$ & $<0.001$ & 71.6 & 0.66 & No \\
\hline $\begin{array}{l}\text { Major adverse cardiovascular and } \\
\text { cerebrovascular events (MACCE) }\end{array}$ & Mahmoud et al. (8) & $\begin{array}{l}3 \text { cohort studies, } \\
4 \text { case-control studies }\end{array}$ & 163,482 & 24,329 & $\mathrm{HR}$ & $1.42(1.26-1.60)$ & $<0.001$ & $<0.001$ & 40 & 0.87 & No \\
\hline Angina & Sacco et al. (9) & $\begin{array}{l}4 \text { cohort studies, } \\
1 \text { cross-sectional study }\end{array}$ & 195,905 & 20,443 & $\mathrm{RR}$ & $1.29(1.17-1.43)$ & $<0.001$ & 0.337 & 12.1 & 0.286 & No \\
\hline Myocardial infarction (MI) & Sacco et al. (9) & $\begin{array}{l}5 \text { cohort studies, } \\
1 \text { case-control study, } \\
1 \text { cross-sectional study }\end{array}$ & 543,810 & 211,589 & $\mathrm{RR}$ & $1.33(1.08-1.64)$ & 0.007 & $<0.001$ & 78.1 & 0.286 & No \\
\hline Ischemic heart disease (IHD) & Sacco et al. (9) & 3 cohort studies & 75,097 & 19,984 & $\mathrm{RR}$ & $1.48(0.94-2.33)$ & 0.091 & $<0.001$ & 92.6 & 0.286 & No \\
\hline Coronary revascularization & Sacco et al. (9) & 3 cohort studies & 48,829 & 6,794 & $\mathrm{RR}$ & $1.11(0.87-1.40)$ & 0.404 & 0.069 & 62.6 & 0.286 & No \\
\hline Cervical artery dissection (CAD) & Rist et al. (28) & 5 case-control studies & 1,315 & 630 & OR & $2.06(1.33-3.19)$ & 0.001 & 0.061 & 55.5 & 0.14 & No \\
\hline $\begin{array}{l}\text { Carotid artery intima-media thickness } \\
\text { (CIMT) }\end{array}$ & Wang et al. (29) & 7 case-control studies & 555 & 279 & SMD & $0.84(0.22,1.45)$ & 0.008 & $<0.001$ & 62.39 & NA & NA \\
\hline $\begin{array}{l}\text { Mean blood flow velocity (MBFV) in the } \\
\text { anterior circulation }\end{array}$ & Dzator et al. (30) & 30 case-control studies & 4,410 & 2,357 & SMD & $0.14(0.05,0.23)$ & 0.003 & $<0.001$ & 47 & NA & NA \\
\hline $\begin{array}{l}\text { Mean blood flow velocity (MBFV) in the } \\
\text { posterior circulation }\end{array}$ & Dzator et al. (30) & 18 case-control studies & 3,145 & 1,855 & SMD & $0.20(0.05,0.34)$ & 0.007 & $<0.001$ & 68 & NA & NA \\
\hline $\begin{array}{l}\text { Pulsatility index (PI) in the anterior } \\
\text { circulation }\end{array}$ & Dzator et al. (30) & 12 case-control studies & 1,406 & 656 & SMD & $-0.02(-0.16,0.13)$ & 0.83 & 0.05 & 36 & NA & NA \\
\hline $\begin{array}{l}\text { Pulsatility index }(\mathrm{PI}) \text { in the posterior } \\
\text { circulation }\end{array}$ & Dzator et al. (30) & 5 case-control studies & 858 & 336 & SMD & $0.23(0.05,0.42)$ & 0.01 & 0.08 & 38 & NA & NA \\
\hline $\begin{array}{l}\text { Cerebrovascular responsiveness (CVR) to } \\
\text { hypercapnia in the anterior circulation }\end{array}$ & Dzator et al. (30) & 26 case-control studies & 2,103 & 1,166 & SMD & $0.11(-0.13,0.35)$ & 0.37 & $<0.001$ & 85 & NA & NA \\
\hline $\begin{array}{l}\text { Cerebrovascular responsiveness (CVR) to } \\
\text { hypercapnia in the posterior circulation }\end{array}$ & Dzator et al. (30) & 11 case-control studies & 1,685 & 991 & SMD & $-0.34(-0.67,-0.01)$ & 0.04 & $<0.001$ & 89 & NA & NA \\
\hline $\begin{array}{l}\text { Cerebrovascular responsiveness (CVR) to } \\
\text { hypocapnia in the anterior circulation }\end{array}$ & Dzator et al. (30) & 8 case-control studies & 352 & 157 & SMD & $0.01(-0.43,0.46)$ & 0.95 & $<0.001$ & 74 & NA & NA \\
\hline $\begin{array}{l}\text { Neurovascular coupling during photic } \\
\text { stimulation in the posterior circulation }\end{array}$ & Dzator et al. (30) & 8 case-control studies & 372 & 220 & SMD & $0.20(-0.15,0.55)$ & 0.26 & 0.03 & 59 & NA & NA \\
\hline Cerebral autoregulation assessed by gain & Dzator et al. (30) & 6 case-control studies & NA & NA & SMD & $-0.21(-0.43,0.01)$ & 0.06 & NA & NA & NA & NA \\
\hline Cerebral autoregulation assessed by phase & Dzator et al. (30) & 6 case-control studies & NA & NA & SMD & $0.13(-0.11,0.36)$ & 0.29 & NA & NA & NA & NA \\
\hline Cerebral autoregulation assessed by $\mathrm{Mx}$ & Dzator et al. (30) & 6 case-control studies & NA & NA & SMD & $0.05(-0.34,0.44)$ & 0.8 & NA & NA & NA & NA \\
\hline
\end{tabular}


TABLE 1 | Continued

\begin{tabular}{|c|c|c|c|c|c|c|c|c|c|c|c|}
\hline Outcomes & References & Number of studies & $\begin{array}{l}\text { Number of } \\
\text { participants }\end{array}$ & $\begin{array}{l}\text { Number of } \\
\text { cases }\end{array}$ & $\begin{array}{l}\text { Type of } \\
\text { metric }\end{array}$ & $\begin{array}{l}\text { Relative risk } \\
\text { (95\% Cl) }\end{array}$ & $\underset{\star}{\mathrm{P} \text { value }}$ & P value \# & $I^{2}(\%)$ & P value* & $\begin{array}{l}\text { Whether exist } \\
\text { publication bias }\end{array}$ \\
\hline Cerebral autoregulation assessed by Dx & Dzator et al. (30) & 6 case-control studies & NA & NA & SMD & $0.29(-0.08,0.66)$ & 0.12 & NA & NA & NA & NA \\
\hline \multicolumn{12}{|l|}{ Imaging abnormalities } \\
\hline White matter abnormalities (WMAs) & $\begin{array}{l}\text { Swartz and Kern } \\
\text { (31) }\end{array}$ & 7 case-control studies & 629 & 312 & OR & $3.90(2.26-6.72)$ & $<0.001$ & 0.66 & 34 & $0.209^{\&}$ & No \\
\hline Infarct-like lesions (ILLs) & Bashir et al. (32) & 2 case-control studies & 3,905 & 522 & OR & $1.07(0.87-1.33)$ & 0.543 & 0.23 & 30.7 & NA & NA \\
\hline Retinal nerve fiber layer (RNFL) thickness & Lin et al. (33) & 26 case-control studies & 2,635 & 1,530 & SMD & $-0.53(-0.75,-0.32)$ & $<0.001$ & $<0.001$ & 85.5 & NA & NA \\
\hline \multicolumn{12}{|l|}{ Pregnancy-linked conditions } \\
\hline Preeclampsia (PE) & Aukes et al. (34) & $\begin{array}{l}3 \text { cohort studies, } \\
6 \text { case-control studies }\end{array}$ & 73,892 & 6,799 & OR & $2.07(1.51-2.85)$ & $<0.001$ & $<0.001$ & 76 & 0.066 & Yes \\
\hline Lowbirth weight (LBW) & Aukes et al. (34) & $\begin{array}{l}2 \text { cohort studies, } \\
1 \text { case-control study }\end{array}$ & 69,031 & 5,888 & OR & $1.18(1.03-1.34)$ & 0.02 & 0.34 & 9 & 0.86 & No \\
\hline Preterm birth (PTB) & Aukes et al. (34) & $\begin{array}{l}3 \text { cohort studies, } \\
2 \text { case-control studies }\end{array}$ & 72,394 & 6,460 & OR & $1.23(0.97-1.55)$ & 0.09 & 0.04 & 61 & 0.337 & No \\
\hline Gestational age (SGA) & Aukes et al. (34) & 2 cohort studies & 30,151 & 5,175 & OR & $1.06(0.98-1.15)$ & 0.14 & 0.47 & 0 & NA & NA \\
\hline \multicolumn{12}{|l|}{ Metabolic conditions } \\
\hline $\begin{array}{l}\text { Low-density lipoprotein cholesterol } \\
(\text { LDL-C) }\end{array}$ & Liampas et al. (35) & $\begin{array}{l}11 \text { case-control } \\
\text { studies, } \\
1 \text { cross-sectional study }\end{array}$ & 2,585 & 1,370 & $\mathrm{MD}$ & $10.44(1.64,19.23)$ & 0.02 & $<0.001$ & 91 & NA & NA \\
\hline $\begin{array}{l}\text { High-density lipoprotein cholesterol } \\
\text { (HDL-C) }\end{array}$ & Liampas et al. (35) & 14 case-control studies & 2,816 & 1,488 & $\mathrm{MD}$ & $-0.37(-2.21,1.47)$ & 0.69 & $<0.001$ & 70 & NA & NA \\
\hline Total cholesterol (TC) & Liampas et al. (35) & $\begin{array}{l}13 \text { case-control } \\
\text { studies, } \\
1 \text { cross-sectional study }\end{array}$ & 2,538 & 1,325 & $\mathrm{MD}$ & $10.56(1.80,19.31)$ & 0.02 & $<0.001$ & 85 & NA & NA \\
\hline Triglycerides(TG) & Liampas et al. (35) & 15 case-control studies & 2,788 & 1,526 & $\mathrm{MD}$ & $11.80(3.62,19.98)$ & 0.005 & $<0.001$ & 67 & NA & NA \\
\hline \multicolumn{12}{|l|}{ Other medical conditions } \\
\hline Phosphene & Brigo et al. (36) & $\begin{array}{l}10 \text { observational } \\
\text { studies }\end{array}$ & 330 & 252 & OR & $3.57(1.16-10.94)$ & 0.03 & 0.01 & 60 & $0.109 \&$ & No \\
\hline Restless legs syndrome (RLS) & Wang et al. (37) & 11 case-control studies & 6,484 & 4,425 & OR & $3.77(2.73-5.21)$ & $<0.001$ & 0.029 & 50.1 & 0.07 & Yes \\
\hline Epilepsy & Keezer et al. (38) & 6 cohort studies & $3,945,421$ & NA & PR & $1.79(1.43-2.25)$ & $<0.001$ & $<0.001$ & 80.8 & NA & NA \\
\hline Breast cancer & Wu et al. (39) & $\begin{array}{l}3 \text { cohort studies, } \\
4 \text { case-control studies }\end{array}$ & 162,954 & 17,776 & $\mathrm{RR}$ & $0.78(0.66-0.92)$ & 0.003 & $<0.001$ & 91.2 & 0.051 & No \\
\hline Infant colic & Zhang et al. (40) & $\begin{array}{l}3 \text { cohort studies, } \\
4 \text { case-control studies }\end{array}$ & 2,935 & 606 & OR & $2.51(1.32-4.77)$ & 0.005 & $<0.001$ & 86 & $0.597^{\&}$ & No \\
\hline Suicidal ideation & Friedman et al. (41) & $\begin{array}{l}5 \text { cross-sectional } \\
\text { studies }\end{array}$ & 148,977 & NA & OR & $2.49(2.34-2.65)$ & $<0.001$ & NA & NA & 0.385 & No \\
\hline $\begin{array}{l}\text { Sudden sensorineural hearing loss } \\
\text { (SSNHL) }\end{array}$ & $\begin{array}{l}\text { Mohammadi et al. } \\
\text { (18) }\end{array}$ & 3 cohort studies & 282,250 & 56,450 & $\mathrm{HR}$ & $1.84(1.11-2.57)$ & $<0.001$ & 0.31 & 76.8 & NA & NA \\
\hline Asthma & Wang et al. (42) & $\begin{array}{l}3 \text { case-control studies, } \\
4 \text { cross-sectional } \\
\text { studies }\end{array}$ & 395,584 & 156,530 & OR & $1.54(1.34-1.77)$ & $<0.001$ & $<0.001$ & 93 & 0.531 & No \\
\hline
\end{tabular}


$2 \quad 2 \quad \frac{\pi}{z}$

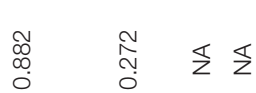

z $\stackrel{2}{z} \stackrel{0}{z}$

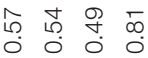

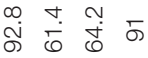

\begin{tabular}{llll} 
V & 0 & 0 & 0 \\
\hdashline & 0 & 0 & 0 \\
0 & 0 & 0
\end{tabular}

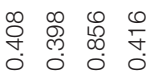

응 $\overline{0}$ 우

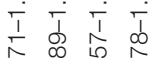

$\stackrel{\circ}{\ominus} \stackrel{\infty}{0} \stackrel{0}{0}$

व.

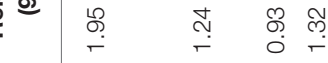

¿

豙

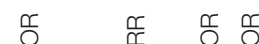

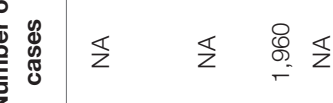

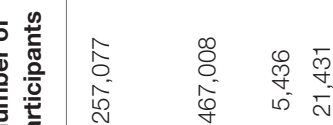

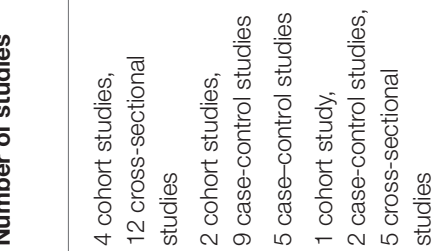

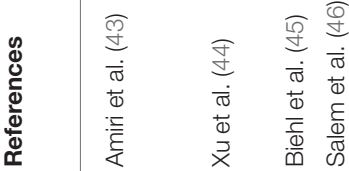
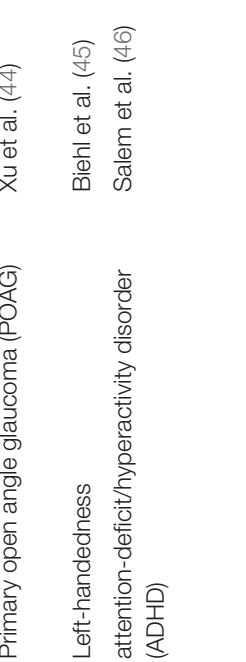

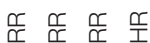

$\Sigma \Sigma \Sigma \frac{8}{8}$

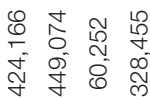

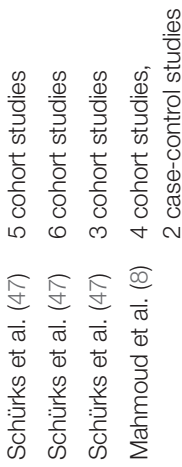

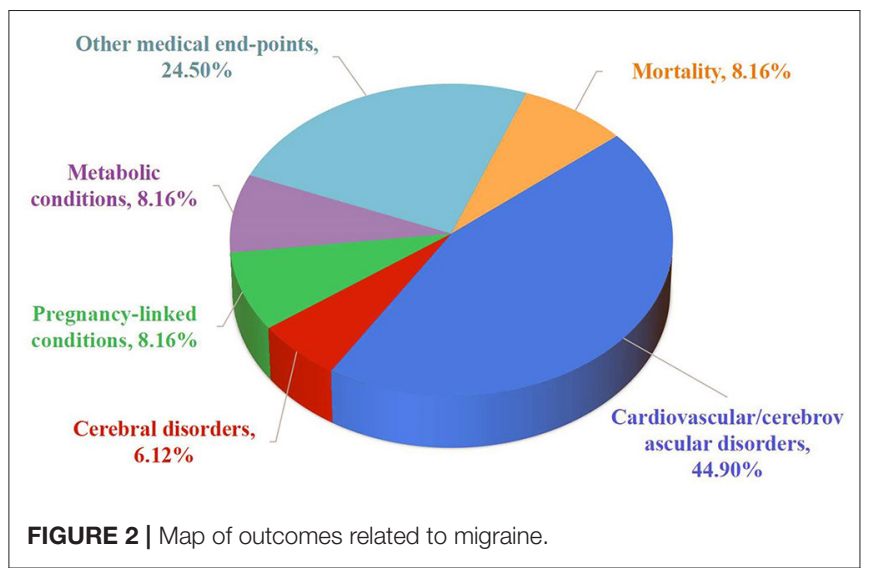

(30). Changes in these indicators confirm that migraine sufferers experience altered cerebrovascular faculties. There was no obvious significant association of migraine with ischaemic heart disease (IHD) and coronary revascularization (9). In addition, none of the variations (between migraineurs and controls) within the following parameters were statistically significant: anterior-circulatory PI variations; CVR to anterior-circulatory hypercapnia/hypocapnia; neurovascular coupling during photic stimulation within posterior circulation; gain-evaluated cerebral autoregulation, phase-evaluated cerebral autoregulation; Mx-evaluated cerebral autoregulation; Dx-evaluated cerebral autoregulation (30).

\section{Imaging Abnormalities}

Migraine was related to an increased risk of white-matter abnormalities (WMAs) on magnetic resonance images (31). Compared to healthy controls, migraineurs demonstrated RNFL hypotrophy (33). However, the meta-analysis of infarct-like lesions (ILLs) on magnetic resonance images showed no association for migraineurs, when compared to controls (32).

\section{Pregnancy-Linked Conditions}

One systematic review and meta-analysis assessed possible associations between migraine and adverse pregnancy medical end-points (34). The results showed that migraine was significantly correlated to elevated risk of preeclampsia (PE) and low birth weight (LBW), though not preterm birth (PTB) and gestational age (SGA).

\section{Metabolic Conditions}

One systematic review and meta-analysis investigated and quantified variations in serum lipid concentrations for both migraineurs/healthy controls (35). Higher low-density lipoprotein cholesterol (LDL-C), cholesterol (TC) and triglyceride (TG) levels were found in migraineurs. The variation in high-density lipoprotein cholesterol (HDL-C) level was not statistically significant.

\section{Other Medical Conditions}

Except for left-handedness (45), associations were found between migraine and the increased risk of phosphene (36), RLS 


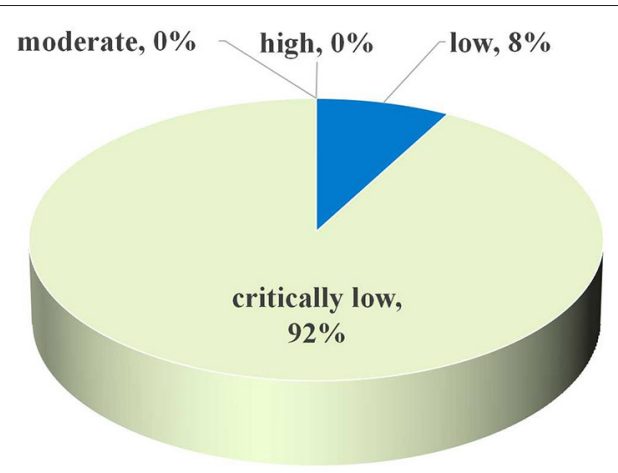

FIGURE 3 | Map of results of AMSTAR 2: percentage of outcomes per outcome category for 25 meta-analyses.

(37), epilepsy (38), infant colic (40), suicidal contemplations (41), SSNHL (18), asthma (42), depression (43), primary open angle glaucoma (POAG) (44), together with attentiondeficit/hyperactivity disorder (ADHD) (46). One meta-analysis reported a statistically significant inverse association between migraine and total breast cancer risk (39).

\section{Mortality}

Surprisingly, although previous studies have shown that migraine has adverse effects on multiple heath medical end-points, it was not associated with mortality from cardiovascular and cerebrovascular (8), cardiovascular (CVD) (47), coronary heart disease (CHD) (47), and all-causes (47).

\section{Heterogeneity}

Among the included meta-analyses, 33\% had very high heterogeneity, $47 \%$ had moderate-to-high heterogeneity, and 6\% had low heterogeneity. However, the remaining 14\% did not report any heterogeneity, and this could not be re-analyzed in this study due to raw data unavailability.

\section{AMSTAR 2 and Summary of Evidence}

Regarding assessment of methodological quality, only two (8\%) investigations were rated as low, with the other 23 (92\%) investigations rated as critically low (Figure 3). This suggested that no single investigation was deemed to carry moderate or high quality, according to AMSTAR2 standards. Following quality-ofproof for every medical end-point, $\sim 80 \%$ were determined to be "weak" and $17 \%$ to be "moderate," only $3 \%$ were determined to be "high" (Figure 4). Detailed information concerning AMSTAR2 and grading of evidence assessments is shown in Tables 2, 3.

\section{DISCUSSION}

This umbrella review identified 49 unique health medical end-points from 25 studies. The results provided a broad overview of the current evidence of relationships between migraine and various health medical end-points, including cardiovascular/cerebrovascular disorders, cerebral disorders, pregnancy-linked conditions, metabolic conditions, other

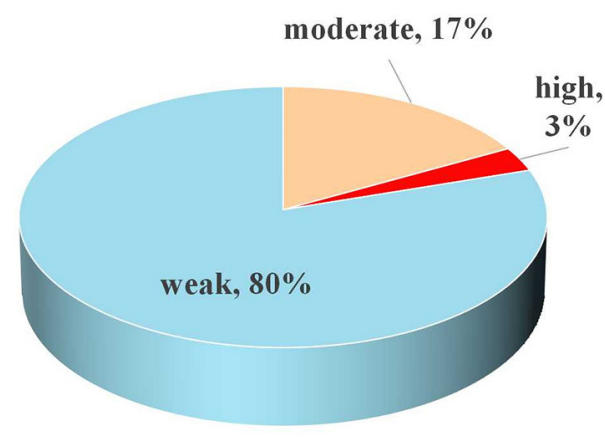

FIGURE 4 | Map of results of evidence assessment: percentage of outcomes per outcome category for 30 meta-analyses.

medical end-points, and mortality. Among these, 30 metaanalyses registered statistically significant results, whereby migraine was linked to reduced breast cancer risk and an increased risk of 29 other medical end-points. However, the evidence quality was graded as high only for angina. The evidence quality of ischaemic stroke, stroke, MACCE, WMAs, and asthma was graded as moderate, while the remaining 24 medical end-points had an evidence grade of "weak."

The International Classification of Headache Diseases (ICHD) has discerned between migraine with aura (MA) and migraine without aura (MO) based on the presence/absence of spreading oligemia (48). The similarities and differences of pathophysiologic, epidemiologic, and clinical proof between migraine with/without aura were reviewed in early studies. Migraine, particularly MA, correlated with exacerbated risk for ischemic/hemorrhagic stroke events (49, 50). The subgroup investigations of ischemic stroke, hemorrhagic stroke, stroke, MI, angina, MBFV in the anterior circulation, RNFL thickness, and phosphene within this umbrella review, showed similar results. Migraine is also $2-3 \times$ fold more prevalent in women (51). Although many studies show no difference in mean pain intensity between men and women, headacherelated disability is reported more frequently in women (52-54). Results of the gender-specific subgroup analyses in this umbrella review showed that the risks of ischemic stroke, hemorrhagic stroke, MI, and angina were elevated in female migraineurs. Cohort study was not greatly influenced through recall/selection biases and was less prone to bias through reverse causality, in comparison to case-control/cross-sectional investigation (55). Correlations between migraine and disease can lead to differing results, depending upon study design. For example, there was a statistically significant inverse association between migraine breast cancer event risk. However, such an inverse relationship was recognized within case-control investigations, though not within cohort investigations. This was consistent with the results of another study (56), which was excluded from this umbrella review. Consequently, larger quantities of prospective cohort studies are required to verify such a correlation. 
TABLE 2 | Detail of results for AMSTAR 2 assessing

References

AMSTAR 2 checklist

Overall assessment

quality

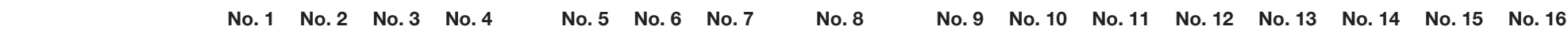

Spector et al. (26) Yes No Yes Yes Yes Yes Partial yes Partial yes\# Yes No Yes Yes Yes No Yes Yes Critically low

Sacco et al. (9) Yes No Yes Partial yes ${ }^{\star}$ Yes Yes Yes

Rist et al. (28) Yes No Yes Partial yes ${ }^{*}$ Yes Yes Yes

Wang et al. (29) Yes No Yes Partial yes* Yes Yes No

Dzator et al. (30) Yes No Yes Partial yes ${ }^{*}$ No No No

Swartz and Kern (31) Yes No Yes No No No No

Bashir et al. (32) Yes No Yes No No No No

Lin et al. (33) Yes Yes Yes Partial yes ${ }^{*}$ No No No

Aukes et al. (34) Yes No Yes Partial yes ${ }^{\star}$ Yes Yes No

Liampas et al. (35) Yes No Yes Yes No No Yes

Brigo et al. (36) Yes No Yes Yes Yes Yes Yes

Wang et al. (37) Yes No Yes Partial yes ${ }^{*}$ Yes Yes No

Keezer et al. (38) Yes No Yes Yes Yes Yes Yes

Wu et al. (39) Yes No Yes Partial yes* Yes Yes No

Zhang et al. (40) Yes No Yes Partial yes ${ }^{*}$ Yes Yes No

Friedman et al. (41) Yes No Yes Partial yes ${ }^{*}$ No No No

Mohammadi et al. (18) Yes No Yes Partial yes ${ }^{\star}$ Yes Yes No

Wang et al. (42) Yes No Yes Partial yes ${ }^{*}$ Yes Yes No

Amiri et al. (43) Yes Yes Yes

Xu et al. (44) Yes No Yes

Biehl et al. (45) Yes No Yes No No No No

Partial yes* ${ }^{*}$ No

Partial yes* Yes

lest Yes

No Yes

Partial yes\# Yes no Yes

Partial yes\# No No Yes

Partial yes\# No No

Partial yes\# Yes No Yes

Partial yes\# Yes No

Partial yes\# Yes No Yes

Partial yes\# No

Partialyes\# Yes No Yes Yes Yes

Partial yes\# Yes no

Partial yes\# No No Yes

Partial yes\# Yes No Yes

Partial yes\# Yes No Yes

Partial yes\# Yes No Yes

Partial yes\# Yes No

Partial yes Partial yes\# Yes No

Salem et al. (46) Yes No Yes No No No No

Partial yes\# Yes

No No No

Schürks et al. (47)

Partial yes $^{*}$ No No No

Partial yes\# No No Yes No

"Partial yes" should meet the following requirements: (1) Search at least 2 databases related to the research question; (2) Provide keywords and/or search strategies; and (3) Explain the restrictions on literature publication, such as language restrictions. In item 8: Did the review authors describe the included studies in adequate detail? "Partial yes\#" should meet the following requirements: (1) Describe the study population; (2) Describe the intervention; (3) Describe control measures; (4) Description of outcome indicators; and (5) Describe the type of study. 
TABLE 3 | Detail of results for evidence quality assessing.

\begin{tabular}{|c|c|c|c|c|c|c|}
\hline \multirow[t]{2}{*}{ Outcomes } & \multirow[t]{2}{*}{ References } & \multicolumn{2}{|c|}{ Precision of the estimate } & \multirow{2}{*}{$\begin{array}{l}\text { Consistency of results } \\
\left(I^{2}<50 \% \text { and Cochran }\right. \\
Q \text {-test } P>0.10)\end{array}$} & \multirow{2}{*}{$\begin{array}{l}\begin{array}{l}\text { No evidence of } \\
\text { small-study effects }\end{array} \\
(P>0.10)\end{array}$} & \multirow[t]{2}{*}{ Grade } \\
\hline & & $\begin{array}{l}>1,000 \text { disease } \\
\text { cases }\end{array}$ & $P<0.001$ & & & \\
\hline \multicolumn{7}{|l|}{$\begin{array}{l}\text { Cardiovascular/cerebrovascular } \\
\text { disorders }\end{array}$} \\
\hline Stroke & Mahmoud et al. (8) & Yes & Yes & No & Yes & Moderate \\
\hline $\begin{array}{l}\text { Major adverse cardiovascular and } \\
\text { cerebrovascular events (MACCE) }\end{array}$ & Mahmoud et al. (8) & Yes & Yes & No & Yes & Moderate \\
\hline Angina & Sacco et al. (9) & Yes & Yes & Yes & Yes & High \\
\hline Myocardial infarction (MI) & Sacco et al. (9) & Yes & No & No & Yes & Weak \\
\hline $\begin{array}{l}\text { Mean blood flow velocity (MBFV) in the } \\
\text { posterior circulation }\end{array}$ & Dzator et al. (30) & Yes & No & No & No & Weak \\
\hline $\begin{array}{l}\text { Pulsatility index (PI) in the posterior } \\
\text { circulation }\end{array}$ & Dzator et al. (30) & No & No & No & No & Weak \\
\hline $\begin{array}{l}\text { Cerebrovascular responsiveness (CVR) } \\
\text { to hypercapnia in the posterior } \\
\text { circulation }\end{array}$ & Dzator et al. (30) & No & No & No & No & Weak \\
\hline \multicolumn{7}{|l|}{ Imaging abnormalities } \\
\hline White matter abnormalities (WMAs) & Swartz and Kern (31) & No & Yes & Yes & Yes & Moderate \\
\hline $\begin{array}{l}\text { Retinal nerve fiber layer (RNFL) } \\
\text { thickness }\end{array}$ & Lin et al. (33) & Yes & Yes & No & No & Weak \\
\hline Triglycerides (TG) & Liampas et al. (35) & Yes & No & No & No & Weak \\
\hline \multicolumn{7}{|l|}{ Other medical conditions } \\
\hline Phosphene & Brigo et al. (36) & No & no & no & yes & Weak \\
\hline Restless legs syndrome (RLS) & Wang et al. (37) & Yes & yes & No & No & Weak \\
\hline Epilepsy & Keezer et al. (38) & No & Yes & No & No & Weak \\
\hline Breast cancer & Wu et al. (39) & Yes & No & No & No & Weak \\
\hline Infant colic & Zhang et al. (40) & No & No & No & Yes & Weak \\
\hline Suicidal ideation & Friedman et al. (41) & No & yes & No & Yes & Weak \\
\hline $\begin{array}{l}\text { Sudden sensorineural hearing loss } \\
\text { (SSNHL) }\end{array}$ & Mohammadi et al. (18) & Yes & yes & No & No & Weak \\
\hline Asthma & Wang et al. (42) & Yes & Yes & No & Yes & Moderate \\
\hline Depression & Amiri et al. (43) & No & Yes & No & Yes & Weak \\
\hline Primary open angle glaucoma (POAG) & Xu et al. (44) & No & Yes & No & Yes & Weak \\
\hline $\begin{array}{l}\text { attention-deficit/hyperactivity disorder } \\
\text { (ADHD) }\end{array}$ & Salem et al. (46) & No & No & No & No & Weak \\
\hline
\end{tabular}

Migraine was associated with 30 medical end-points. However, serious heterogeneity between studies existed in most of the meta-analyses. The following factors contributed to the heterogeneity of the included meta-analyses: age, geographical area, migraines ascertainment, migraine aura status, and study design. The standards of methods implemented in all 
selected meta-analyses was categorized as "critically low" or "low," mostly because of a "no" decision on the following items: a pre-recognized explicit statement/protocol, a list of excluded studies, bias risk assessment in selected studies, funding source details for the selected studies, discussion of heterogeneity observed within review results, report of potential sources for conflicts of interest. Only one medical end-point was rated as high quality-of-proof. Many studies did not report results for $I^{2}$ statistic, $P$-value for Cochran's $Q$-test, and $P$-value for Egger's test, leading to a decline in evidence grade.

The authors believe this is a pioneering investigational effort to assess properly all links between migraine and multiple health/medical end-points through adoption of an umbrella review approach. The authors performed a critical appraise of the range and validity of reported relationships between migraine and diverse health/medical end-points. Notwithstanding, some limitations inevitably existed in this umbrella review. Firstly, results of individual observational investigations involving under-developed meta-analysis were beyond the scope of this review, such as the concentration of lipoprotein(a) (57) and diabetes (58). Thus, we might have missed some researches on the links between migraine and multiple health/medical endpoints. Secondly, when two or more meta-analyses reported identical health/medical end-points, the report containing the most studies was selected, regardless of study design. Therefore, the results may be skewed by the influence of recall/selection bias and reverse causality. Thirdly, one study reported that migraine increased the risk of IBS, though this umbrella review did not select this study since the full-text was not available. We tried to contact the author to obtain the full text, but failed, which resulted in the loss of a very important research result. Fourthly, this umbrella review did not include publications in languages other than English. The link between migraines and health/medical end-points reported in other languages may have been overlooked. Consequently, conclusion bias of association

\section{REFERENCES}

1. Silberstein SD. Migraine. Lancet. (2004) 363:38191. doi: 10.1016/S0140-6736(04)15440-8

2. Dodick DW. A phase-by-phase review of migraine pathophysiology. Headache. (2018) 58(Suppl. 1):4-16. doi: 10.1111/head.13300

3. Lambru G, Benemei S, Andreou AP, Luciani M, Serafini G, van den Brink AM, et al. Position paper on post-traumatic headache: the relationship between head trauma, stress disorder, and migraine. Pain Ther. (2021) 10:1-13. doi: 10.1007/s40122-020-0 0220-1

4. Yeh WZ, Blizzard L, Taylor BV. What is the actual prevalence of migraine? Brain Behav. (2018) 8:e00950. doi: 10.1002/brb3.950

5. Takeshima T, Wan Q, Zhang Y, Komori M, Stretton S, Rajan N, et al. Prevalence, burden, and clinical management of migraine in China, Japan, and South Korea: a comprehensive review of the literature. J Headache Pain. (2019) 20:111. doi: 10.1186/s10194-019-1062-4

6. Schürks M, Rist PM, Bigal ME, Buring JE, Lipton RB, Kurth T. Migraine and cardiovascular disease: systematic review and meta-analysis. BMJ. (2009) 339:b3914. doi: 10.1136/bmj.b3914 between migraine and human health can be produced by the aforementioned situations.

In conclusion, this review provided a detailed evaluation of all available data on links between migraine and various health/medical end-points. The results showed that migraine increased the risk of 29 health/medical end-points and reduced the risk of breast cancer. Considering that evidence for most medical end-points were categorized as "moderate" and "weak," additional high-quality prospective cohort studies are required in order to draw a firm conclusion.

\section{DATA AVAILABILITY STATEMENT}

The original contributions presented in the study are included in the article/supplementary material, further inquiries can be directed to the corresponding author.

\section{AUTHOR CONTRIBUTIONS}

WC: idea, design, and manuscript revision. WQ and GJ: literature search, data extraction, and analysis. LZ: manuscript writing. All authors read and approved the version of the manuscript to be published and took responsibility for appropriate content.

\section{FUNDING}

This study was supported by 2021 Zunyi Science and Technology Bureau and Zunyi First People's Hospital joint science and Technology Research and development fund project [Zun Kehe HZ Word (2021) No. 233].

\section{ACKNOWLEDGMENTS}

We would like to thank the researchers and study participants for their contributions.
7. Hu X, Zhou Y, Zhao H, Peng C. Migraine and the risk of stroke: an updated meta-analysis of prospective cohort studies. Neurol Sci. (2017) 38:3340. doi: 10.1007/s10072-016-2746-z

8. Mahmoud AN, Mentias A, Elgendy AY, Qazi A, Barakat AF, Saad M, et al. Migraine and the risk of cardiovascular and cerebrovascular events: a metaanalysis of 16 cohort studies including 1152407 subjects. BMJ Open. (2018) 8:e020498. doi: 10.1136/bmjopen-2017-020498

9. Sacco S, Ornello R, Ripa P, Tiseo C, Degan D, Pistoia F, et al. Migraine and risk of ischaemic heart disease: a systematic review and meta-analysis of observational studies. Euro J Neurol. (2015) 22:100111. doi: 10.1111/ene.12701

10. Denuelle M, Fabre N, Payoux P, Chollet F, Geraud G. Posterior cerebral hypoperfusion in migraine without aura. Cephalalgia. (2008) 28:85662. doi: 10.1111/j.1468-2982.2008.01623.x

11. Larrosa-Campo D, Ramón-Carbajo C, Álvarez-Escudero R, CernudaMorollón E, García-Cabo C, Pascual J. Arterial pathology in migraine: endothelial dysfunction and structural changes in the brain and systemic vasculature. Rev Neurol. (2015) 61:313-22. doi: 10.33588/rn.6107.2015226

12. Tietjen GE. Migraine as a systemic vasculopathy. Cephalalgia. (2009) 29:98796. doi: 10.1111/j.1468-2982.2009.01937.x 
13. Tietjen GE, Collins SA. Hypercoagulability and migraine. Headache. (2018) 58:173-83. doi: 10.1111/head.13044

14. Yang X, Liu B, Yang B, Li S, Wang F, Li K, et al. Prevalence of restless legs syndrome in individuals with migraine: a systematic review and meta-analysis of observational studies. Neurol Sci. (2018) 39:192734. doi: 10.1007/s10072-018-3527-7

15. Burch RC, Rist PM, Winter AC, Buring JE, Pradhan AD, Loder EW, et al. Migraine and risk of incident diabetes in women: a prospective study. Cephalalgia. (2012) 32:991-7. doi: 10.1177/0333102412453954

16. Wongtrakul W, Charoenngam N, Ungprasert P. Increased prevalence of irritable bowel syndrome in migraine patients: a systematic review and meta-analysis. Eur $J$ Gastroenterol Hepatol. (2021). doi: 10.23736/S2724-5985.21.02957-0

17. Feng YF, Guo H, Huang JH Yu JG, Yuan F. Retinal nerve fiber layer thickness changes in migraine: a meta-analysis of case-control studies. Curr Eye Res. (2016) 41:814-22. doi: 10.3109/02713683.2015.1056373

18. Mohammadi M, Taziki Balajelini MH, Rajabi A. Migraine and risk of sudden sensorineural hearing loss: a systematic review and meta-analysis. Laryngoscope Invest Otolaryngol. (2020) 5:1089-95. doi: 10.1002/lio2.477

19. Dresler T, Caratozzolo S, Guldolf K, Huhn JI, Loiacono C, Niiberg-Pikksööt $\mathrm{T}$, et al. Understanding the nature of psychiatric comorbidity in migraine: a systematic review focused on interactions and treatment implications. $J$ Headache Pain. (2019) 20:51. doi: 10.1186/s10194-019-0988-x

20. Shamseer L, Moher D, Clarke M, Ghersi D, Liberati A, Petticrew M, et al. Preferred reporting items for systematic review and meta-analysis protocols (PRISMA-P) 2015: elaboration and explanation. BMJ. (2015) 350:g7647. doi: 10.1136/bmj.g7647

21. Altman DG, Bland JM. How to obtain the confidence interval from a P value. BMJ. (2011) 343:d2090. doi: 10.1136/bmj.d2090

22. Shea BJ, Reeves BC, Wells G, Thuku M, Hamel C, Moran J, et al. 2: a critical appraisal tool for systematic reviews that include randomised or non-randomised studies of healthcare interventions, or both. BMJ. (2017) 358:j4008. doi: 10.1136/bmj.j4008

23. Piovani D, Danese S, Peyrin-Biroulet L, Nikolopoulos GK, Lytras T, Bonovas S. Environmental risk factors for inflammatory bowel diseases: an umbrella review of meta-analyses. Gastroenterology. (2019) 157:64759.e4. doi: 10.1053/j.gastro.2019.04.016

24. Tsilidis KK, Kasimis JC, Lopez DS, Ntzani EE, Ioannidis JP. Type 2 diabetes and cancer: umbrella review of meta-analyses of observational studies. BMJ. (2015) 350:g7607. doi: 10.1136/bmj.g7607

25. Chen W, Li Y, Guo L, Zhang C, Tang S. An umbrella review of systematic reviews and meta-analyses of observational investigations of obstructive sleep apnea and health outcomes. Sleep Breath. (2021). doi: 10.1007/s11325-021-02384-2. [Epub ahead of print].

26. Spector JT, Kahn SR, Jones MR, Jayakumar M, Dalal D, Nazarian S. Migraine headache and ischemic stroke risk: an updated meta-analysis. Am J Med. (2010) 123:612-24. doi: 10.1016/j.amjmed.2009.12.021

27. Sacco S, Ornello R, Ripa P, Pistoia F, Carolei A. Migraine and hemorrhagic stroke: a meta-analysis. Stroke. (2013) 44:30328. doi: 10.1161/STROKEAHA.113.002465

28. Rist PM, Diener HC, Kurth T, Schürks M. Migraine, migraine aura, and cervical artery dissection: a systematic review and meta-analysis. Cephalalgia. (2011) 31:886-96. doi: 10.1177/0333102411401634

29. Wang Q, Liu ZY, Zhou J. Ultrasonic assessment of carotid intima-media thickness in migraine: a meta-analysis. J Int Med Res. (2019) 47:284855. doi: 10.1177/0300060519851354

30. Dzator JS, Howe PR, Wong RH. Profiling cerebrovascular function in migraine: a systematic review and meta-analysis. J Cereb Blood Flow Metab. (2021) 41:919-44. doi: 10.1177/0271678X20964344

31. Swartz RH, Kern RZ. Migraine is associated with magnetic resonance imaging white matter abnormalities: a meta-analysis. Arch Neurol. (2004) 61:13668. doi: 10.1001/archneur.61.9.1366

32. Bashir A, Lipton RB, Ashina S, Ashina M. Migraine and structural changes in the brain: a systematic review and meta-analysis. Neurology. (2013) 81:12608. doi: 10.1212/WNL.0b013e3182a6cb32

33. Lin X, Yi Z, Zhang X, Liu Q, Zhang H, Cai R, et al. Retinal nerve fiber layer changes in migraine: a systematic review and meta-analysis. Neurol Sci. (2021) 42:871-81. doi: 10.1007/s10072-020-04992-4
34. Aukes AM, Yurtsever FN, Boutin A, Visser MC, de Groot CJM. Associations between migraine and adverse pregnancy outcomes: systematic review and meta-analysis. Obstetr. Gynecol. Survey. (2019) 74:738-48. doi: 10.1097/OGX.0000000000000738

35. Liampas I, Mylonas KS, Brotis A, Dervenis P, Siokas V, Mentis AA, et al. Serum lipid abnormalities in migraine: a meta-analysis of observational studies. Headache. (2021) 61:44-59. doi: 10.1111/head.14039

36. Brigo F, Storti M, Tezzon F, Manganotti P, Nardone R. Primary visual cortex excitability in migraine: a systematic review with meta-analysis. Neurol Sci. (2013) 34:819-30. doi: 10.1007/s10072-012-1274-8

37. Wang J, Gao Y, Liu L, Xu W, Zhang P, Liu Y, et al. The association between migraine and restless legs syndrome: an updated systematic review and meta-analysis. Sleep Med. (2019) 57:21-9. doi: 10.1016/j.sleep.2019.01.027

38. Keezer MR, Bauer PR, Ferrari MD, Sander JW. The comorbid relationship between migraine and epilepsy: a systematic review and meta-analysis. Euro J Neurol. (2015) 22:1038-47. doi: 10.1111/ene.12612

39. Wu X, Wang $\mathrm{M}$, Li S, Zhang $\mathrm{Y}$. Migraine and breast cancer risk: a meta-analysis of observational studies based on MOOSE compliant. Medicine. (2016) 95:e4031. doi: 10.1097/MD.00000000000 04031

40. Zhang D, Zhang Y, Sang Y, Zheng N, Liu X. The relationship between infant colic and migraine as well as tension-type headache: a metaanalysis. Pain Res Manag. (2019) 2019:8307982. doi: 10.1155/2019/83 07982

41. Friedman LE, Gelaye B, Bain PA, Williams MA. A systematic review and meta-analysis of migraine and suicidal ideation. Clin J Pain. (2017) 33:65965. doi: 10.1097/AJP.0000000000000440

42. Wang L, Deng ZR, Zu MD, Zhang J, Wang Y. The comorbid relationship between migraine and asthma: a systematic review and meta-analysis of population-based studies. Front Med. (2020) 7:609528. doi: 10.3389/fmed.2020.609528

43. Amiri S, Behnezhad S, Azad E. Migraine headache and depression in adults: a systematic review and meta-analysis. Neuropsychiatr. (2019) 33:13140. doi: 10.1007/s40211-018-0299-5

44. Xu C, Li J, Li Z, Mao X. Migraine as a risk factor for primary open angle glaucoma: a systematic review and meta-analysis. Medicine. (2018) 97:e11377. doi: 10.1097/MD.0000000000011377

45. Biehl K, Frese A, Marziniak M, Husstedt IW, Evers S. Migraine and lefthandedness are not associated. A new case-control study and meta-analysis. Cephalalgia. (2008) 28:553-7. doi: 10.1111/j.1468-2982.2008.01553.x

46. Salem H, Vivas D, Cao F, Kazimi IF, Teixeira AL, Zeni CP, et al. is associated with migraine: a systematic review and meta-analysis. Eur Child Adolesc Psychiatry. (2018) 27:267-77. doi: 10.1007/s00787-017-1045-4

47. Schürks M, Rist PM, Shapiro RE, Kurth T. Migraine and mortality: a systematic review and meta-analysis. Cephalalgia. (2011) 31:130114. doi: $10.1177 / 0333102411415879$

48. Vgontzas A, Burch R. Episodic migraine with and without aura: key differences and implications for pathophysiology, management, assessing risks. Curr Pain Headache Rep. (2018) 22:78. doi: 10.1007/s11916-018-0735-z

49. Scher AI, Terwindt GM, Picavet HS, Verschuren WM, Ferrari MD, Launer LJ. Cardiovascular risk factors and migraine: the GEM population-based study. Neurology. (2005) 64:614-20. doi: 10.1212/01.WNL.0000151857.43 225.49

50. Yemisci M, Eikermann-Haerter K. Aura and stroke: relationship and what we have learnt from preclinical models. J Headache Pain. (2019) 20:63. doi: 10.1186/s10194-019-1016-x

51. Vetvik KG, MacGregor EA. Sex differences in the epidemiology, clinical features, and pathophysiology of migraine. Lancet Neurol. (2017) 16:7687. doi: 10.1016/S1474-4422(16)30293-9

52. Burch R, Rizzoli P, Loder E. The prevalence and impact of migraine and severe headache in the united states: figures and trends from government health studies. Headache. (2018) 58:496-505. doi: 10.1111/head.13281

53. GBD 2016 Headache Collaborators. Global, regional, and national burden of migraine and tension-type headache, 1990-2016: a systematic analysis for the Global Burden of Disease Study 2016. Lancet Neurol. (2018) 17:95476. doi: 10.1016/S1474-4422(18)30322-3

54. Steiner TJ, Scher AI, Stewart WF, Kolodner K, Liberman J, Lipton RB. The prevalence and disability burden of adult migraine in England and 
their relationships to age, gender and ethnicity. Cephalalgia. (2003) 23:51927. doi: $10.1046 / j .1468-2982.2003 .00568 . x$

55. Yi M, Wu X, Zhuang W, Xia L, Chen Y, Zhao R, et al. Tea consumption and health outcomes: umbrella review of meta-analyses of observational studies in humans. Mol Nutr Food Res. (2019) 63:e1900389. doi: 10.1002/mnfr.201900389

56. Winter AC, Rice MS, Fortner RT, Eliassen AH, Kurth T, Tamimi RM. Migraine and breast cancer risk: a prospective cohort study and meta-analysis. J Natl Cancer Inst. (2015) 107:381. doi: 10.1093/jnci/d ju381

57. Mattiuzzi C, Cervellin G, Lippi G. Epidemiological association between migraine and lipoprotein(a): a systematic review. $J$ Thromb Thrombolysis. (2015) 39:113-7. doi: 10.1007/s11239-014-1 079-0

58. Hosseinpour M, Maleki F, Khoramdad M, Sullman MJM, Nejadghaderi SA, Kolahi AA, et al. systematic literature review of observational studies of the bilateral association between diabetes and migraine. Diabetes Metab Syndr. (2021) 15:673-8. doi: 10.1016/j.dsx.2021.03.018
Conflict of Interest: The authors declare that the research was conducted in the absence of any commercial or financial relationships that could be construed as a potential conflict of interest.

Publisher's Note: All claims expressed in this article are solely those of the authors and do not necessarily represent those of their affiliated organizations, or those of the publisher, the editors and the reviewers. Any product that may be evaluated in this article, or claim that may be made by its manufacturer, is not guaranteed or endorsed by the publisher.

Copyright $\odot 2021$ Chen, Qian, Zhong and Jing. This is an open-access article distributed under the terms of the Creative Commons Attribution License (CC BY). The use, distribution or reproduction in other forums is permitted, provided the original author(s) and the copyright owner(s) are credited and that the original publication in this journal is cited, in accordance with accepted academic practice. No use, distribution or reproduction is permitted which does not comply with these terms. 